\title{
Unconscious processing of color and form in metacontrast masking
}

\author{
Tony Ro \\ City College of New York, CUNY, New York \\ NeEL S. Singhal \\ University of Pennsylvania, Philadelphia, Pennsylvania \\ Bruno G. BREITMEYER \\ University of Houston, Houston, Texas \\ AND \\ JAVIER O. GARCIA \\ University of California, Irvine, California
}

\begin{abstract}
Three experiments employed a metacontrast masking procedure to examine the extent and nature of priming effects from visual stimuli not consciously perceived. The results showed effects of unconscious stimuli on subsequent target responses that (1) were more consistent, reliable, and not subject to strategic control, as compared with consciously perceived stimuli (Experiment 1); (2) produced both facilitation and interference of subsequent processing (Experiment 2); and (3) did not influence indirect response-related levels of processing (Experiment 3 ). These results demonstrate that color and form attributes of unconscious stimuli are sufficiently registered within the visual system to influence behavior, and that some of these unconscious effects occur at early levels of stimulus encoding, prior to higher level perceptual and response-related processes.
\end{abstract}

While performing a given task, such as making a cup of tea, one can be distracted by all sorts of things; a misplaced pan, for example, can cause a delay in, and modification of, the plan of action. Often these distractions go unnoticed, but they may nonetheless automatically activate and alter the perceptual and motor systems, especially when certain stimulus features, such as the color or shape of objects, are shared. The role of visual awareness in generating facilitation and inhibition of subsequent perceptual and response processing was investigated in this study using a metacontrast masking paradigm that allowed the presentation of stimuli below a conscious threshold for awareness (for a review on metacontrast masking, see Breitmeyer \& Öğmen, 2000). The main question addressed was whether or not a stimulus has to be consciously registered in order for it to have an influence on other stimuli and, particularly, at what levels this unconscious influence occurs.

As early as the 1950s and '60s, studies showed a dissociation between phenomenal reports of observers and their physiological and overt responses (Fehrer \& Biederman, 1962; Fehrer \& Raab, 1962; Lazarus \& McCleary, 1951). For example, Fehrer and her colleagues demonstrated that when participants were asked to respond with a speeded buttonpress to the first stimulus seen, they responded faster when there was a preceding metacontrast masked stimulus, despite reporting having been unaware of it This speeded response suggests that, although observers often do not report awareness of the masked stimuli, these stimuli may initiate a motor response.

More recently, the role of awareness in perceptual and response-related processing has been the subject of much interest and debate. Since the elegant studies by Marcel (1983) demonstrating unconscious semantic priming, numerous studies have examined whether unconscious visual stimuli influence the perceptual and response-related processing of other stimuli. In one of these studies, Schwarz and Mecklinger (1995) used a backward pattern-masking procedure and demonstrated that, although participants were unable to consciously report the identity (objective thresholding procedure) of flanking letter distractors, they nonetheless produced slower responses when these flankers were incongruent with the target letter. Similar results have been reported using a metacontrast masking procedure with shapes (Klotz \& Neumann, 1999) and colors (Schmidt, 2000, 2002), and have shown that unconscious stimuli may automatically influence actions (Schmidt, 2002; Vorberg, Mattler, Heinecke, Schmidt, \& Schwarzbach, 2003). Other studies, using patterned masks, have also shown influences on subsequent responses from unconscious, semantically related stimuli

T. Ro, tro@ccny.cuny.edu 
(Abrams, Klinger, \& Greenwald, 2002; Dehaene et al., 1998; Greenwald, Draine, \& Abrams, 1996; Klinger, Burton, \& Pitts, 2000), including numeric stimuli assigned to shared response mappings (Naccache, Blandin, \& Dehaene, 2002). An event-related potential study suggests that these unconscious semantic processing effects reflect automatic spreading of activation to semantically related stimuli, possibly in the ventral visual pathway (Kiefer, 2002; Kiefer \& Brendel, 2006).

Studies in neurological patients also suggest the presence of influences from distractors presented outside of awareness. Previous results in patients with hemispatial neglect (for reviews on neglect, see Driver \& Mattingley, 1998; Rafal, 2000) - who have deficits in orienting toward, and are typically unaware of, contralesional events - have generally demonstrated that a normal flanker interference effect occurs from stimuli flanking central targets in the contralesional "unaware" hemifield of these patients (Audet, Bub, \& Lecours, 1991; Cohen, Ivry, Rafal, \& Kohn, 1995; Snow \& Mattingley, 2006). However, in the study by Audet et al., one of the two neglect patients failed to demonstrate any interference effects from contralesional distractors. Furthermore, in a study investigating the effects of lesions on flanker interference, we found that patients with neglect initially failed to demonstrate any effects from contralesional colored flankers if their lesion involved the temporoparietal junction (Ro, Cohen, Ivry, \& Rafal, 1998). Given these inconsistencies, one goal of the present series of experiments was to measure the consistency of perceptual processing in the absence of awareness in neurologically intact participants.

A set of studies in neurologically normal participants has also provided some inconsistent evidence regarding the effects of unconsciously processed primes on later target responses. Using arrowhead-like stimuli (symbols meaning greater than and less than), Eimer and colleagues have demonstrated that incongruent pattern masked stimuli can, under certain conditions, produce a facilitation, rather than an inhibition, in responses (Eimer, 1999; Eimer \& Schlaghecken, 1998; for a review of this work, see Eimer \& Schlaghecken, 2003). These studies by Eimer and Schlaghecken, showing a different, but not incompatible, pattern of results from unconscious stimuli, also used objective thresholding procedures. The differences in results between these studies may be due to many factors, including the different types of stimuli and masks used (Lleras \& Enns, 2004), the way that threshold for awareness was measured between these studies (Cheesman \& Merikle, 1986; Holender, 1986; Holender \& Duscherer, 2004; Snodgrass, Bernat, \& Shevrin, 2004), and/or the time interval separating prime presentation and response execution.

In an attempt to resolve some of these inconsistencies, a color discrimination task was used in the first two experiments of this study to demonstrate effects on perceptual and response-related processing without awareness, while using the simplest stimuli possible that varied on only one surface feature dimension. In each of the present experiments, we assessed awareness by subjective reports from participants on a trial-by-trial basis. Judging by the findings from one of the two patients by Audet et al. (1991), and by both patients in the study by Cohen et al. (1995), as well as those obtained from most of the studies in neurologically intact participants, we expected that an effect on color and form processing would be measured in neurologically normal participants, despite unawareness of a preceding event. However, in a manner consistent with previous studies on unconscious processing, the magnitude of this effect might be different from the magnitude measured with awareness (Cheesman \& Merikle, 1986; Dagenbach, Carr, \& Wilhelmsen, 1989). In particular, unconscious colored stimuli might have greater and more reliable influences on subsequent processing than consciously perceived ones, since consciously perceived stimuli could invoke strategies and biases that might interact with stimulus processing (Dagenbach et al., 1989). This was the particular focus of Experiment $1 .{ }^{1}$

Additionally, it remains to be determined whether the effects of unconscious stimuli facilitate, inhibit, or both facilitate and inhibit the processing of other visual stimuli. Therefore, by using a neutral colored stimulus, ${ }^{2}$ Experiment 2 assessed the level at which these unconscious color processing effects take place. Finally, although it has been suggested that unconscious color stimuli affect response channel activation (Audet et al., 1991; Cohen et al., 1995; Ro et al., 1998; Schmidt, 2000, 2002), this issue has only indirectly been addressed in previous studies. Thus, Experiment 3 used shapes, rather than colors, with different combinations of response assignments, to extend the results from the previous experiments examining unconscious perceptual and response priming and to more precisely determine whether response channel activation occurs without awareness.

\section{EXPERIMENT 1}

In previous experiments, it has been shown that the processing of many different stimulus attributes can occur in the absence of awareness. However, it might be that the interference/priming measured in those and previous experiments in both patients (Audet et al., 1991; Cohen et al., 1995) and neurologically intact participants (Eimer \& Schlaghecken, 2003; Klotz \& Neumann, 1999; Schwarz \& Mecklinger, 1995) was due to the small proportion of trials in which the participants were aware of the supposed unconscious stimulus. Conversely, it is possible that unperceived stimuli might produce more reliable interference/ priming than consciously perceived ones, which may be subject to conscious strategies and biases that reduce or inhibit the influences of these stimuli. In this experiment, we attempted to address these issues by asking participants after each trial whether or not they were aware of the disk that preceded the annulus.

\section{Method}

Participants. Eighteen young, neurologically normal students (12 females, 6 males) participated in this experiment after giving informed consent. The participants were recruited from Rice University and received course credit for participating in a single session. Two of the participants did not have a sufficient number of trials in the unaware condition; the participants reported awareness of the 
Stimuli

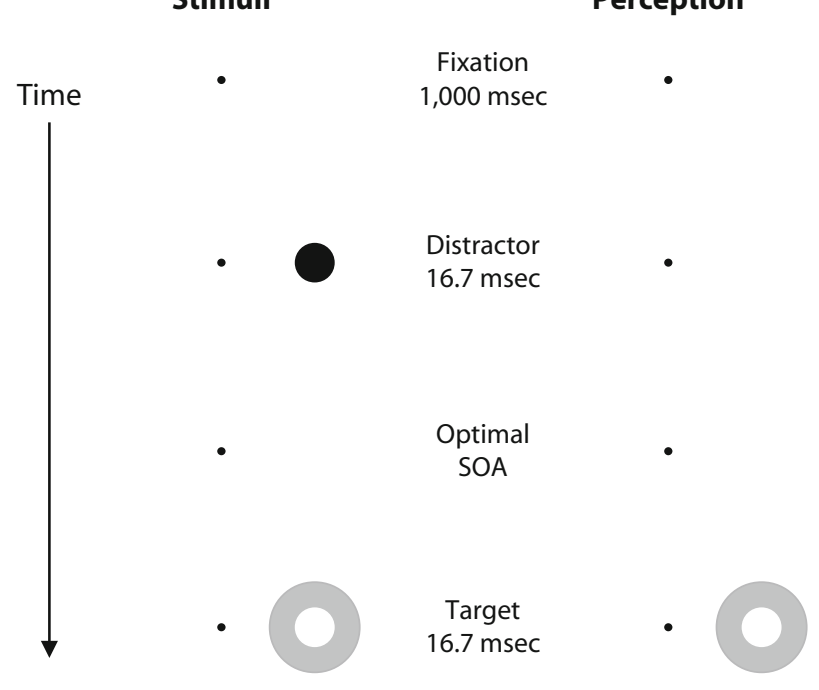

Figure 1. The sequence of events on a typical incongruent trial for Experiments 1 and 2. The left column depicts the actual sequence of events, whereas the right column depicts the observer's perception of the events.

masked stimulus on a majority of the trials. Only the data from the remaining 16 participants were used. The mean age of the 16 participants was 19.8 years (age range, $17-32$ years).

Apparatus. This experiment was conducted on an IBM-compatible personal computer connected to a Sony Trinitron VGA stimulus monitor. The timing of the visual displays was controlled by the vertical synchronization of the stimulus monitor at $16.67-\mathrm{msec}$ intervals $(60 \mathrm{~Hz})$. Millisecond timing, used to obtain response latencies, was achieved by setting the 8253 chip of the computer to millisecond ticks. Responses were made on a two-button response pad connected to the gameport adapter of the computer. Response times (RTs) were recorded to the nearest millisecond, following a buttonpress.

Stimuli and Procedures. The stimuli in this experiment consisted of a fixation point, a colored disk, and a colored annulus, all presented on a black background. The luminance of the blue and green colors used in this experiment was adjusted for each participant, so that the perceived intensities of these colors were the same. This was accomplished before the experiment by using heterochromatic flicker fusion at $30 \mathrm{~Hz}$ with $1^{\circ}$ disks placed $3^{\circ}$ to the left or right. While participants fixated the point at the center of the screen, the intensity of the blue disk was adjusted to the green disk until the flicker was minimized. Four intensity settings were obtained, then averaged independently for the left and right sides.

After the isoluminant settings for each participant were determined, a $0.1^{\circ}$ light gray fixation point was presented at the center of the screen for the duration of the experiment. On each trial, either a blue or a green disk appeared $3^{\circ}$ to the left or right of fixation followed by a blue or green annulus $57 \mathrm{msec}$ later. The disk was $1^{\circ}$ in diameter and was presented for $16.7 \mathrm{msec}$, whereas the annulus was $2^{\circ}$ in diameter with an inner diameter of $1^{\circ}$. The annulus was also presented for $16.7 \mathrm{msec}$ (see Figure 1). The color (blue or green) and location (left or right) of the disk and annulus were both randomly selected with equal probability, with the constraint that both the disk and annulus appeared in the same location. The location of the disk and annulus, both either left or right, was manipulated on the basis of a recent report demonstrating larger masking effects with location varied stimuli (Enns \& Di Lollo, 1997). The disk and the annulus colors were either identical (both blue or both green) or incongruent (one blue and one green) to one another with equal probability.

Participants were seated $57 \mathrm{~cm}$ from the computer monitor in a diffusely lit room. They were instructed to respond to the annulus color by pressing one button on the response pad if the color was blue, and the other button on the response pad if the color was green. For half the participants, the left button indicated a blue annulus and the right button indicated a green annulus; for the other half, the assignments were reversed. The participants were instructed to respond as fast and accurately as possible. The trial was terminated if no response was made by $2,000 \mathrm{msec}$ after the onset of the annulus. Once a color buttonpress response had been made, the screen went blank, and the participants were asked to verbally indicate whether they had perceived the disk in that trial. After the awareness response had been recorded by the experimenter, presentation of the next trial began.

Design. Responses were collected from each of the 2 awareness $\times 2$ congruency conditions, until there was a minimum of 20 trials for each of the four conditions. Because the incongruent disks with respect to the annuli were not as easily masked as the congruent disks were, there were typically many more unaware trials for the congruent conditions.

\section{Results}

All responses faster or slower than $2 S D$ s from the mean and incorrect keypress responses were discarded. These error trials comprised $7.81 \%$ of all trials and are broken down by condition in Table 1 , along with the mean number of trials per condition. Figure 2 shows the RT results for the correct response trials in the annulus report task.

Mean RTs from the correct trials were subjected to a two-way ANOVA with awareness (aware vs. unaware) and congruency (congruent vs. incongruent) as the two within-participants factors. We used means instead of medians because of the unequal proportion of trials completed by each participant (Miller, 1988). This analysis revealed no significant main effects (both $F \mathrm{~s}<1$ ) but a significant awareness $\times$ congruency interaction $[F(1,15)=$

Table 1

Mean Response Times (RTs, in Milliseconds), Number of Trials Performed Across Participants, and Percentages of Errors in Experiment 1

\begin{tabular}{lcrrrrrr}
\hline & \multicolumn{3}{c}{ Aware } & & \multicolumn{3}{c}{ Unaware } \\
\cline { 2 - 4 } \cline { 6 - 8 } Congruency & RT & Trials & PE & & RT & Trials & PE \\
\hline Congruent & 698.3 & 73.0 & 7.7 & & 727.2 & 222.7 & 7.5 \\
Incongruent & 725.3 & 212.2 & 8.4 & & 696.1 & 83.0 & 7.2 \\
\hline
\end{tabular}

Note-Included in the errors are incorrect responses and RTs greater or less than $2 S D$ s from the mean.

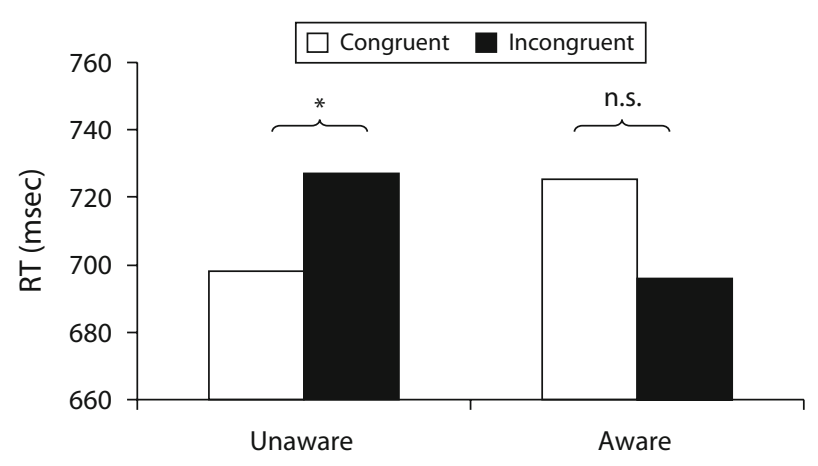

Awareness Condition

Figure 2. Mean response times (RTs) from Experiment 1 for the different masking conditions. $\quad * p<.05$. 
5.42, $p<.05]$. To determine the source of this interaction, paired $t$ tests were performed separately on the aware and unaware responses. For the unaware conditions, the interference/priming from the colored disks on the subsequent annulus was significant, with slower responses being generated in the incongruent conditions $[t(15)=$ $2.53, p<.025]$. The congruency effect in the aware trials did not achieve significance $(p>.15)$.

\section{Discussion}

These results add to the body of evidence in normal participants, suggesting that perceptual processing can occur in the absence of awareness (Eimer \& Schlaghecken, 2003; Klotz \& Neumann, 1999; Schwarz \& Mecklinger, 1995 ) and provide converging evidence with the results reported by Cohen et al. (1995) demonstrating flanker interference effects from contralesional distractors in patients with hemispatial neglect. More importantly, this experiment demonstrates a difference in performance between consciously perceived and unperceived stimuli. As with previous studies, unconscious colored stimuli produced reliable interference/priming, with unconscious incongruent disks increasing RTs to the subsequent annulus mask. However, the conscious preceding disk stimuli produced no reliable effects on subsequent annulus processing. In fact, the overall pattern of results was the opposite of typical interference/priming effects, with faster responses for incongruent trials and slower responses for congruent trials. This difference in results between the two awareness conditions was obtained despite the stimuli and procedures being identical across these conditions.

One possible source of this opposite pattern of results for the aware conditions may have been due to a conscious response strategy that participants may have adopted in the aware conditions. Since participants were three times as likely to consciously perceive the disk when it was incongruent than when it was congruent (see Table 1), participants might have learned this stimulus-stimulus contingency and used a strategic response bias. If so, participants might have prepared an opposite response when the preceding disk was consciously perceived, countering the typical congruency effect. Without conscious awareness of the stimulus, however, such a conscious strategy, based on the attributes of the stimulus, would be difficult to implement, and therefore may not have been invoked. A post hoc analysis, including the number of trials completed, suggests that there was in fact a tendency for participants to sometimes adopt this type of strategy at some point early in the experiment. For the 8 participants with sufficient trials for this analysis, ${ }^{3}$ Figure 3 shows the RTs for the congruent and incongruent trials in the aware conditions plotted for the first two sets of at least four trials each that were completed (i.e., each set comprised a sixteenth of the total number of trials completed). As can be seen in this figure, performance was initially similar to the performance in the unaware trials, with slower responses in the incongruent conditions. However, in the second sixteenth of trials, the pattern of results was reversed, with slower responses for the congruent trials. The proportion of trials completed $\times$ congruency interac-

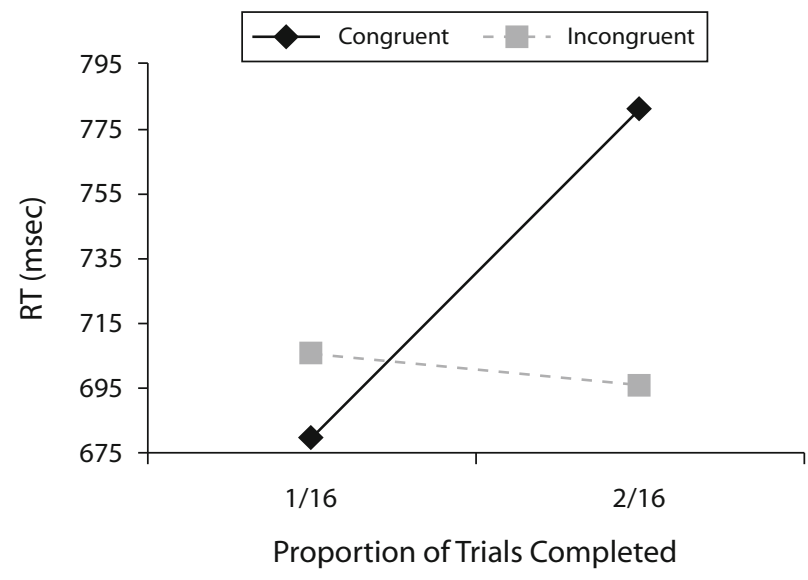

Figure 3. Mean response times (RTs) from Experiment 1 for the first two sixteenths of trials in the aware conditions. Note the opposite RT pattern between the two time segments of the trials, suggestive of a learning strategy that the participants were applying.

tion for the aware trials approached significance $[F(1,7)=$ $3.87, p=.09]$. To further assess whether this reverse congruency effect ever achieved significance, analyses on the second sixteenth, as well as on the second half of the trials, were conducted, but they were not significant, perhaps because of the counteracting effects from the conscious versus unconscious processing systems. Nonetheless, this suggests that this stimulus-stimulus contingency may have been learned and utilized by some participants, allowing for some preparation of the opposite color response and, hence, faster incongruent RTs when the disk was consciously perceived. When the mask was congruent with the disk, however, participants had to veto this prepared response, causing a delay in the congruent, aware condition. The response preparation based on this strategy likely contributed to the overall slower aware trials in this experiment.

Additionally, it is possible that the task in this experiment, which had the additional requirement of asking participants after each trial whether or not the disk had been perceived, contributed to the inconsistent results with the congruent aware disks. ${ }^{4}$ After each trial, the participants were responsible for retrieving from consciousness any information about the encoding of the disk. It is obvious by the increased RTs in the main task of this experimentcompared with other experiments we have conducted where two responses were not required on each trial - that this additional task requirement increased the processing time necessary for the color response. Perhaps the additional effort of retrieving this information in combination with the inhibitory strategic processes slowed RTs to the aware, congruent stimuli for some of the participants.

Regardless of the mechanisms that eliminated and tended to reverse the effects in the aware conditions, the main results from this experiment demonstrate that color processing occurs in the absence of awareness. They further suggest that, under conditions of awareness, some conscious strategies may be applied that can sometimes override the typical interference/priming effects; but with- 
out awareness of the disks, such a strategy may not be possible, thereby leading to normal interference/priming effects from the disks.

\section{EXPERIMENT 2}

Experiment 1 and other studies investigating the effects of unconscious processing do not indicate whether the unconscious interference/priming effects are due to facilitation, inhibition, or both facilitation and inhibition of subsequent processing. Experiment 2 employed a neutral colored disk that was generated by combining the colors of the two masks. Thus, a green, blue, or cyan disk could appear with equal probability before a green or a blue annulus mask. We expected that the unconscious neutral stimulus in this experiment would produce intermediate RTs, as compared with those for identical or responseincongruent stimuli. In addition to employing a neutral stimulus, this experiment used a stimulus presentation rate - based on objective thresholding procedures from previous experiments (see Breitmeyer, Ro, Öğmen, \& Todd, 2007; Breitmeyer, Ro, \& Singhal, 2004) - that rendered the majority of the masked stimuli unconscious.

\section{Method}

Participants. Twelve young, neurologically normal students ( $7 \mathrm{fe}-$ male, 5 male) participated in this experiment after giving informed consent. None of the individuals had participated in the previous experiment. The participants were recruited from the University of Houston and received course credit for participating in a single session. The mean age of the participants was 24.8 years (age range, 19-32 years).

Stimuli and Procedures. The stimuli and procedures were similar to those in Experiment 1, but with the following exceptions. The stimuli were displayed at a $100-\mathrm{Hz}$ frame rate on a Sony Trinitron color monitor. Stimulus presentation and response recording were controlled by a visual stimulus generator (VSG2/5) card manufactured by Cambridge Research Systems. To maximize the number of unseen disks as opposed to seen disks, the blue, cyan, and green stimuli were desaturated and presented at $16.0 \mathrm{~cd} / \mathrm{m}^{2}$ on an $8.0-\mathrm{cd} / \mathrm{m}^{2}$ gray background. The blue, cyan, and green stimuli had hue saturation value coordinates of $(240.5,0.668,0.289),(109.91,0.783$, $0.157)$, and $(179.87,1,0.153)$, respectively. Because desaturated colors were used, all stimuli were presented in the center of the screen. The prime and mask stimuli were each presented for $10 \mathrm{msec}$ at an optimal metacontrast SOA of $50 \mathrm{msec}$. Since these parameters, along with these colored stimuli, have been demonstrated in previous studies to produce near complete masking (Breitmeyer et al., 2007; Breitmeyer et al., 2004), reports of awareness after each trial were not collected in this experiment.

Design. For each participant, 40 responses were collected from each of the three congruency conditions (congruent, neutral, and incongruent).

\section{Results}

All responses faster than $150 \mathrm{msec}$ and slower than $900 \mathrm{msec}$, as well as incorrect buttonpresses, were discarded from the analysis. ${ }^{5}$ These error trials comprised $4.2 \%$ of all trials and were not further analyzed. Figure 4 shows the RT results for the correct response trials in the annulus report task.

Mean RTs from the correct trials were subjected to a one-way ANOVA with congruency (congruent vs. neutral vs. incongruent) as the within-participants factor.

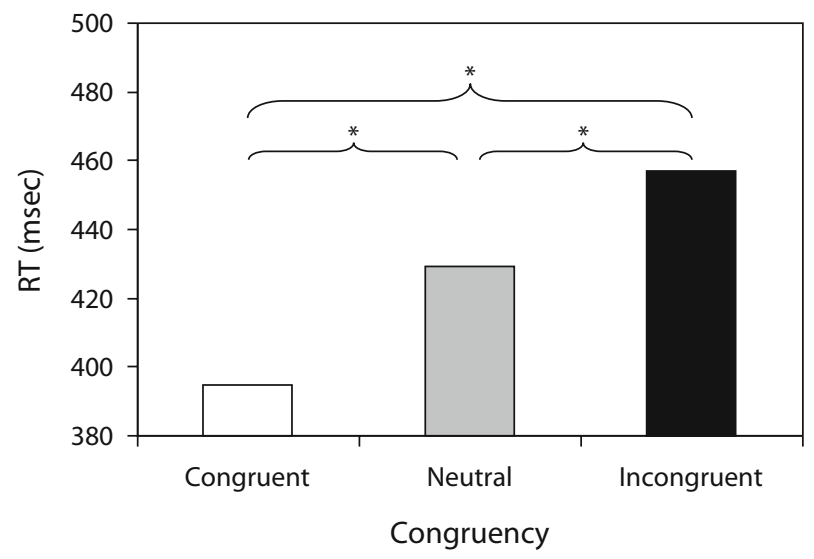

Figure 4. Mean response times (RTs) from Experiment 2 for the different masking conditions. ${ }^{*} p<.05$.

This analysis revealed a significant effect of congruency $[F(2,22)=43.66, p<.0005]$. Paired $t$ tests comparing the neutral condition with the congruent and incongruent condition were performed; they demonstrated that there was a significant facilitation $[t(11)=5.09, p<.001]$, as well as a significant inhibition $[t(11)=2.73, p<.025]$, from congruent and incongruent disks, respectively.

\section{Discussion}

Experiment 2 suggests both a facilitatory and an inhibitory effect of the unconscious colored stimuli. When the unconscious disk was identical in color to the target mask, responses were significantly faster than were those to a neutral colored disk. In addition, when the unconscious disk was of the opposite response (e.g., a green disk followed by a blue mask), there was significant inhibition (i.e., slowing) on subsequent processing from the incongruent disk. These results demonstrate that some aspects of the unconscious interference/priming effects may be due to both facilitation and inhibition.

\section{EXPERIMENT 3}

Experiment 3 was conducted to extend the findings from the previous two experiments, focusing on whether response priming can occur without awareness. Furthermore, instead of using colors, shapes were used to determine whether our results could be extended to other stimulus features in addition to surface property features such as color.

\section{Method}

Participants. Sixteen participants (9 female, 7 male), none of whom had participated in any of the previous experiments, successfully completed this experiment after giving informed consent. The mean age of the participants was 19.2 years (age range, $17-21$ years). The participants were recruited from Rice University and received course credit for participating in a single session. The extremely high error rates of 1 participant $(50 \%)$ caused that participant's data to be discarded.

Stimuli and Procedures. The procedures were similar to those in the previous experiments, except for the following changes. The masks were semicircles in this experiment, and measured $1.5^{\circ}$ in 
radius; they contained an inner circle with crosshairs for more efficient metacontrast masking (see Figure 5A). On every trial, a smaller semicircle measuring $0.5^{\circ}$ in radius preceded the mask by $42 \mathrm{msec}$. The smaller semicircle was positioned to fall completely within the inner circle of the mask and could be, with equal probability, a left, right, lower, or upper semicircle. The larger semicircles (i.e., the masks) could also be, with equal probability, left, right, lower, or upper semicircles. Participants were asked to respond with one button (the left one for half of the participants) to a left semicircle or an upper semicircle (or a right semicircle or a lower semicircle), and with the other button for a right semicircle or a lower semicircle (or a left semicircle or an upper semicircle).

These response mappings produced three main conditions: (1) an identical condition, in which the prime and probe were identical; (2) a congruent-response condition, in which the prime and probe required the same response but were not physically identical; and (3) an incongruent-response condition, in which primes and probes were physically different and required different responses. Because each of the four smaller semicircles could appear equally often with each of the larger semicircles, $25 \%$ of the trials were identical smaller and larger semicircle conditions; another $25 \%$ of the trials were responsecongruent conditions; and the remaining $50 \%$ of the trials were response-incongruent conditions. A total of 640 trials was completed by each participant. After a response was made to the target semicircle, the participants were asked to report whether or not they saw a smaller preceding semicircle. If the unconscious representations of the primes do not influence response-related levels of processing, we should find faster responses only for the identical prime and probe conditions, in comparison with the incongruent conditions; but if unconscious processing also occurs at response-related stages of processing, faster responses should also occur for the congruent-response conditions compared with the incongruent-response conditions.

\section{Results}

Table 2 shows the mean number of correct trials completed by the participants for each of the congruency $X$ awareness conditions, as well as the mean error rates for each of the conditions. The error data, which totaled $8.8 \%$ of the trials and included incorrect button responses, or RTs greater than or equal to $1,500 \mathrm{msec}$, were not further analyzed. Because the majority of the stimuli were unconscious to the participants, we only analyzed the unaware trials, as in Experiment 2. As in the previous studies, RTs to the semicircle masks were influenced by stimuli that preceded them but were unconscious (see Figure 5B). A one-way ANOVA with congruency (identical, congruent response, incongruent response) as the within-participants factor was conducted on the data from trials on which participants were unaware of the preceding semicircle. This analysis revealed a significant main effect of congruency $[F(2,28)=19.3, p<.001]$.

Planned comparisons with the incongruent condition were conducted to more precisely determine the sources

Table 2

Mean Number of Correct Trials, and Percentages of Errors, Performed Across Participants in Experiment 3

\begin{tabular}{lccccc}
\hline & \multicolumn{2}{c}{ Aware } & & \multicolumn{2}{c}{ Unaware } \\
\cline { 2 - 3 } \cline { 5 - 6 } Congruency & $M$ & PE & & $M$ & PE \\
\hline Identical & 1.2 & 0.1 & & 140.7 & 11.3 \\
Congruent & 2.6 & 0.0 & & 137.7 & 12.5 \\
Incongruent & 5.5 & 0.0 & & 274.9 & 12.5 \\
\hline
\end{tabular}

A

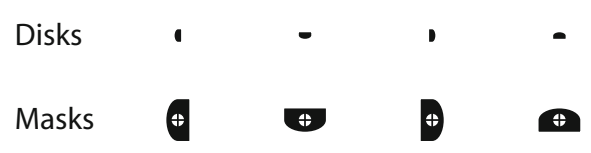

B

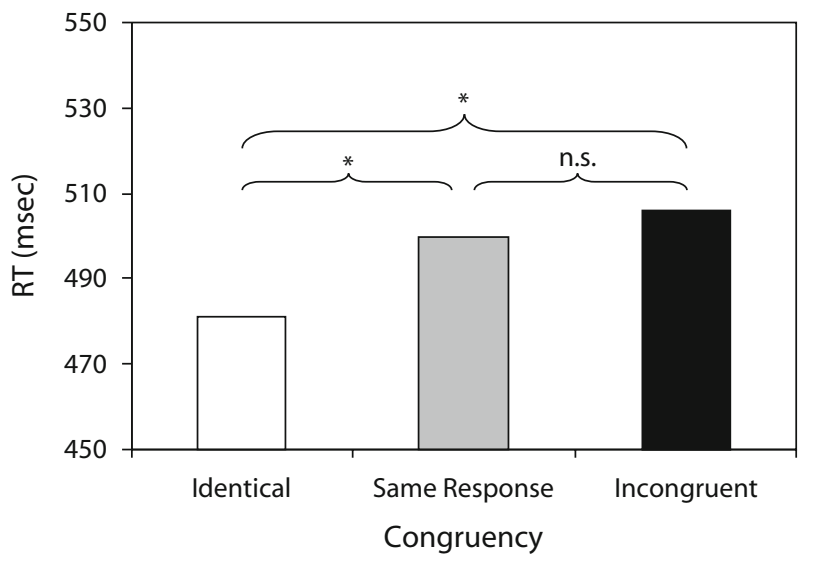

Figure 5. (A) Examples of the stimuli that were used. (B) Mean response times (RTs) from Experiment 3 for the different masking conditions. ${ }^{*} p<.05$.

of this significant main effect. These further analyses showed, as did those in the previous experiments, a significant difference between the identical semicircles and the incongruent semicircles (both physically and by response assignment), with slower RTs in the incongruent conditions $[t(14)=7.9, p<.001]$. However, responses to semicircles following physically different semicircles but requiring the same response were not significantly faster than were physically different semicircle pairs that did not share the same response $(p>.20)$. These results demonstrate that there were no unconscious influences of the stimuli on response-related levels of processing.

\section{Discussion}

This experiment extends the results of Experiments 1 and 2 by showing that response priming does not occur in the absence of awareness, and that some of these unconscious influences of distractors/primes occur at very early, stimulus-dependent levels of processing. Thus, although shapes can be primed without awareness, this experiment shows that response priming does not proceed in the absence of awareness. Whereas this result may seem at odds with other studies demonstrating response priming from unconscious stimuli (Eimer \& Schlaghecken, 1998; Klotz \& Neumann, 1999; Vorberg et al., 2003), note that our study used a more complex design, where more than one stimulus was mapped to the same response, and that only physically identical unconscious stimuli facilitated responses to subsequent stimuli. In the General Discussion section, we discuss other possible explanations for why we did not measure a response priming effect for physically different stimuli with shared responses. 


\section{GENERAL DISCUSSION}

Our results demonstrate that both shape and color can be processed without awareness, and that at least some of these unconscious processing effects occur at early levels of visual processes prior to higher order perceptual and response-related processes. In Experiment 1, we assessed the awareness of the disks after each trial and separately analyzed the magnitude of the effects for the conditions in which participants were and were not aware of the colored disks. The results demonstrated a robust and reliable influence from colored stimuli when participants were unaware of these disks, but not when they were aware of them. In Experiment 2, we showed that unconscious stimuli can produce both facilitation and inhibition of subsequent processes by employing a neutral unconscious stimulus for comparisons. Experiment 3 extended these findings to demonstrate unconscious form perception and confirmed that the unconscious processing of stimuli occurs at early levels of processing, prior to response assignment. Taken together, these results in normals provide converging evidence, along with studies in neurological patients with unilateral deficits in awareness (Audet et al., 1991; Cohen et al., 1995), that unconscious color and form processing occurs in the absence of awareness.

In other studies using metacontrast masking to investigate color priming, Schmidt $(2000,2002)$ reported some findings similar to the ones reported here. In the studies by Schmidt, however, the magnitude of color interference/ priming without awareness was never directly compared with the magnitude of interference/priming with awareness under identical conditions, as in Experiment 1. Furthermore, it was unclear at what processing level these unconscious effects occur, and whether the participants were objectively or subjectively "aware" of the stimuli during the critical trials in which the effects from the unaware stimulus were assessed, since awareness was usually measured in separate blocks of trials. ${ }^{6}$ Thus, our study extends and complements previous findings by providing evidence that perceptual processing does occur in the absence of awareness, and that this interference/priming without awareness is more reliable than that with identically masked distractors that do enter awareness. This more reliable effect from unconscious stimuli is in line with the unconscious semantic priming literature, which shows that, in contrast to consciously perceived words, unconscious words may not be used strategically to perform a given task (Cheesman \& Merikle, 1986; Merikle \& Joordens, 1997; Merikle, Joordens, \& Stolz, 1995). Thus, the semantic activations without awareness most likely reflect basic automatic activations of words without any influences from higher order cognitive processes, such as strategic or voluntary control.

The more reliable effects from masked stimuli outside of awareness were most clearly demonstrated in Experiment 1 , in which we found that incongruent unconscious primes slowed down responses, whereas incongruent conscious primes facilitated them. Although our experiments cannot directly explain why these reversed effects occur with awareness, ${ }^{7}$ these reversed effects may be ac- counted for by the "dissociation-disconnection" theory of unconscious activation (Dagenbach et al., 1989), which was originally postulated to explain unconscious semantic priming. According to this theory, the mask disconnects the further encoding of the disk from consciously controllable strategies, and unperceived disks are processed by automatic perceptual encoding mechanisms. Given the absence of intentional strategies, it is no surprise that the unperceived stimuli in our experiments produced effects on subsequently presented stimuli. However, the opposite results obtained for the consciously perceived disks in the aware conditions of Experiment 3 may be the result of some form of strategic process to inactivate, or invoke, inhibitory mechanisms that may suppress effects automatically generated from these stimuli. As reported previously, priming could be either facilitatory or inhibitory, depending on whether participants are required to make a detection or semantic judgment about a masked word (Dagenbach et al., 1989). This suggests that the mechanisms of stimuli presented at near-threshold levels can be affected by consciously derived strategies for retrieving perceptually encoded information.

Some previous studies investigating unconscious priming have suggested that unaware stimuli directly influence subsequent response-related levels of processing (Eimer \& Schlaghecken, 2003; Klotz \& Neumann, 1999). Furthermore, the majority of these studies have described these unconscious effects as occurring entirely within, or directly influencing, the motor system (but see Lleras $\&$ Enns, 2004, 2006). Most convincingly, some of these studies have shown that the magnitude of these response priming effects is not dependent on the level of prime visibility (Vorberg et al., 2003), and that movement trajectories can be influenced by unconscious stimuli (Schmidt, 2002). Although we agree that there may indeed be direct motor parameter specification, Experiment 3 provides some limits and displays boundary conditions of these unconscious response-related effects. By using stimuli with overlapping and shared response assignments, we provide evidence that unconscious processing only influences stimulus-response pairing with a direct (one-toone) mapping. Thus, the unconscious processing system does not seem able to influence more abstract or complex forms of response-related processes or stimulus-response mappings, at least not with highly unfamiliar stimuli such as those used in Experiment 3 (for somewhat different results using highly familiar stimuli such as numbers or words, see Klinger et al., 2000; Naccache et al., 2002).

In conclusion, the present results demonstrate that the unconscious processing of color and form occurs at relatively early levels of processing, prior to stages that are influenced by strategic control and more abstract representations of stimulus-response mappings. Despite these restricted forms of unconscious representations, these primitive codes may nonetheless influence, in some very limited ways, response-related processing (Klotz \& Neumann, 1999; Schmidt, 2002; Vorberg et al., 2003) and perhaps even decision making (Dijksterhuis, 2004; Dijksterhuis, Bos, Nordgren, \& van Baaren, 2006; Nisbett \& Wilson, 1977). Other related studies from our laboratory 
suggest that manipulations of spatial attention do not affect the magnitude of flanker interference generated (Ro, Machado, Kanwisher, \& Rafal, 2002). Thus, as others have also suggested (Miller, 1991), it appears that flanker interference and priming effects are quite consistent and robust across a number of different physical and cognitive manipulations. Taken together, the results from our studies indicate that cognitive manipulations, such as attention to (Ro et al., 2002) and awareness of (the present experiments) stimuli, do not influence the processing that irrelevant visual stimuli may invoke.

\section{AUTHOR NOTE}

This work was supported in part by US PHS training fellowship MH19930 to T.R., US PHS Grant MH41544 to R. D. Rafal, US PHS Grant MH64606 to T.R., NSF Grant BCS-0114533 to B.B., and NSF Grant BCS-0642801 to T.R. We thank Bob Rafal and Lynn Robertson for their helpful comments and suggestions on earlier experiments of this work, which were completed while T.R. was at the University of California, Davis. We also thank Jose Ramon for assisting with data collection for Experiment 2. Correspondence concerning this article should be addressed to T. Ro, Department of Psychology, NAC 7/120, The City College of the City University of New York, New York, NY 10031 (e-mail: tro@ccny.cuny.edu).

\section{REFERENCES}

Abrams, R. L., Klinger, M. R., \& Greenwald, A. G. (2002). Subliminal words activate semantic categories (not automated motor responses). Psychonomic Bulletin \& Review, 9, 100-106.

Audet, T., Bub, D., \& Lecours, A. R. (1991). Visual neglect and leftsided context effects. Brain \& Cognition, 16, 11-28. doi:10.1016/02782626(91)90082-J

Breitmeyer, B. G., \& ÖĞMen, H. (2000). Recent models and findings in visual backward masking: A comparison, review, and update. Perception \& Psychophysics, 62, 1572-1595.

BreitMeyer, B. G., Ro, T, ÖĞMEN, H., \& TodD, S. (2007). Unconscious, stimulus-dependent priming and conscious, percept-dependent priming with chromatic stimuli. Perception \& Psychophysics, 69, 550-557.

Breitmeyer, B. G., Ro, T., \& Singhal, N. S. (2004). Unconscious color priming occurs at stimulus- not percept-dependent levels of processing. Psychological Science, 15, 198-202. doi:10.1111/j.09567976.2004.01503009.x

Cheesman, J., \& Merikle, P. M. (1986). Distinguishing conscious from unconscious perceptual processes. Canadian Journal of Psychology, 40, 343-367. doi: $10.1037 / \mathrm{h} 0080103$

Cohen, A., Ivry, R. B., Rafal, R. D., \& Kohn, C. (1995). Activating response codes by stimuli in the neglected visual field. Neuropsychology, 9, 165-173. doi:10.1037/0894-4105.9.2.165

Dagenbach, D., Carr, T. H., \& Wilhelmsen, A. (1989). Task-induced strategies and near-threshold priming: Conscious influences on unconscious perception. Journal of Memory \& Language, 28, 412-443. doi:10.1016/0749-596X(89)90020-X

Dehaene, S., Naccache, L., Le Clec'H, G., Koechlin, E., MuelLer, M., Dehaene-Lambertz, G., ET AL. (1998). Imaging unconscious semantic priming. Nature, 395, 597-600. doi:10.1038/26967

Dijksterhuis, A. (2004). Think different: The merits of unconscious thought in preference development and decision making. Journal of Personality \& Social Psychology, 87, 586-598. doi:10.1037/00223514.87.5.586

Dijksterhuis, A., Bos, M. W., Nordgren, L. F., \& van BaAren, R. B (2006). On making the right choice: The deliberation-without-attention effect. Science, 311, 1005-1007. doi:10.1126/science.1121629

Driver, J., \& Mattingley, J. B. (1998). Parietal neglect and visual awareness. Nature Neuroscience, 1, 17-22. doi:10.1038/217

EIMER, M. (1999). Facilitatory and inhibitory effects of masked prime stimuli on motor activation and behavioural performance. Acta Psychologica, 101, 293-313. doi:10.1016/S0001-6918(99)00009-8

Eimer, M., \& SCHLAGHECKEN, F. (1998). Effects of masked stimuli on motor activation: Behavioral and electrophysiological evidence. Jour- nal of Experimental Psychology: Human Perception \& Performance, 24, 1737-1747. doi:10.1037/0096-1523.24.6.1737

Eimer, M., \& Schlaghecken, F. (2003). Response facilitation and inhibition in subliminal priming. Biological Psychology, 64, 7-26. doi:10.1016/S0301-0511(03)00100-5

EnNS, J. T., \& Di Lollo, V. (1997). Object substitution: A new form of masking in unattended visual locations. Psychological Science, $\mathbf{8}$, 135-139. doi:10.1111/j.1467-9280.1997.tb00696.x

FeHrer, E., \& Biederman, I. (1962). A comparison of reaction time and verbal report in the detection of masked stimuli. Journal of Experimental Psychology, 64, 126-130. doi:10.1037/h0044410

FeHrer, E., \& RAAB, D. (1962). Reaction time to stimuli masked by metacontrast. Journal of Experimental Psychology, 63, 143-147. doi:10.1037/h0040795

Greenwald, A. G., Draine, S. C., \& Abrams, R. L. (1996). Three cognitive markers of unconscious semantic activation. Science, 273, 1699-1702. doi:10.1126/science.273.5282.1699

HoLENDER, D. (1986). Semantic activation without conscious identification in dichotic listening, parafoveal vision, and visual masking: A survey and appraisal. Behavioral \& Brain Sciences, 9, 1-66.

Holender, D., \& DUSCherer, K. (2004). Unconscious perception: The need for a paradigm shift. Perception \& Psychophysics, 66, 872-881.

JONIDES, J., \& MACK, R. (1984). On the cost and benefit of cost and benefit. Psychological Bulletin, 96, 29-44. doi:10.1037/0033-2909.96.1.29

Kiefer, M. (2002). The N400 is modulated by unconsciously perceived masked words: Further evidence for an automatic spreading activation account of N400 priming effects. Cognitive Brain Research, 13, 27-39. doi:10.1016/S0926-6410(01)00085-4

Kiefer, M., \& Brendel, D. (2006). Attentional modulation of unconscious "automatic" processes: Evidence from event-related potentials in a masked priming paradigm. Journal of Cognitive Neuroscience, 18, 184-198. doi: $10.1162 / 089892906775783688$

Klinger, M. R., Burton, P. C., \& Pitts, G. S. (2000). Mechanisms of unconscious priming: I. Response competition, not spreading activation. Journal of Experimental Psychology: Learning, Memory, \& Cognition, 26, 441-455. doi:10.1037/0278-7393.26.2.441

Klotz, W., \& NeumanN, O. (1999). Motor activation without conscious discrimination in metacontrast masking. Journal of Experimental Psychology: Human Perception \& Performance, 25, 976-992. doi:10.1037/0096-1523.25.4.976

Lazarus, R. S., \& MCCleary, R. A. (1951). Autonomic discrimination without awareness: A study of subception. Psychological Review, 58, 113-122. doi: $10.1037 / \mathrm{h} 0054104$

Lleras, A., \& ENNS, J. T. (2004). Negative compatibility or object updating? A cautionary tale of mask-dependent priming. Journal of Experimental Psychology: General, 133, 475-493. doi:10.1037/00963445.133.4.475

Lleras, A., \& EnNs, J. T. (2006). How much like a target can a mask be? Geometric, spatial, and temporal similarity in priming: A reply to Schlaghecken and Eimer (2006). Journal of Experimental Psychology: General, 135, 495-500. doi:10.1037/0096-3445.135.3.495

MarCEL, A. J. (1983). Conscious and unconscious perception: Experiments on visual masking and word recognition. Cognitive Psychology, 15, 197-237. doi:10.1016/0010-0285(83)90009-9

MeriKLe, P. M., \& Joordens, S. (1997). Parallels between perception without attention and perception without awareness. Consciousness \& Cognition, 6, 219-236. doi:10.1006/ccog.1997.0310

Merikle, P. M., Joordens, S., \& Stolz, J. A. (1995). Measuring the relative magnitude of unconscious influences. Consciousness \& Cognition, 4, 422-439. doi:10.1006/ccog.1995.1049

Miller, J. (1988). Response-compatibility effects in focused-attention tasks: A same-hand advantage in response activation. Perception \& Psychophysics, 43, 83-89.

MiLLER, J. (1991). The flanker compatibility effect as a function of visual angle, attentional focus, visual transients, and perceptual load: A search for boundary conditions. Perception \& Psychophysics, 49, 270-288.

Naccache, L., Blandin, E., \& Dehaene, S. (2002). Unconscious masked priming depends on temporal attention. Psychological Science, 13, 416-424. doi:10.1111/1467-9280.00474

NisbetT, R. E., \& Wilson, T. D. (1977). Telling more than we can know: Verbal reports on mental processes. Psychological Review, 84, 231259. doi:10.1037/0033-295X.84.3.231

Rafal, R. D. (2000). Neglect II: Cognitive neuropsychological issues 
In M. J. Farah \& T. E. Feinberg (Eds.), Patient-based approaches to cognitive neuroscience (pp. 125-141). Cambridge, MA: MIT Press.

Ro, T., CoHen, A., IVRY, R. B., \& RAFAL, R. D. (1998). Response channel activation and the temporoparietal junction. Brain \& Cognition, 37, 461-476. doi:10.1006/brcg.1998.1008

Ro, T., Machado, L., Kanwisher, N., \& Rafal, R. D. (2002). Covert orienting to the locations of targets and distractors: Effects on response channel activation in a flanker task. Quarterly Journal of Experimental Psychology, 55A, 917-936. doi:10.1080/02724980143000514

Ro, T., \& SingHAL, N. [S.] (2000, November). Distractor interference in the absence of awareness. Poster presented at the 41st Annual Meeting of the Psychonomic Society, New Orleans.

Schmidt, T. (2000). Visual perception without awareness: Priming responses by color. In T. Metzinger (Ed.), Neural correlates of consciousness: Empirical and conceptual questions (pp. 157-169). Cambridge, MA: MIT Press.

SchMidT, T. (2002). The finger in flight: Real-time motor control by visually masked color stimuli. Psychological Science, 13, 112-117. doi:10.1111/1467-9280.00421

Schwarz, W., \& Mecklinger, A. (1995). Relationship between flanker identifiability and compatibility effect. Perception \& Psychophysics, 57, 1045-1052.

Snodgrass, M., Bernat, E., \& Shevrin, H. (2004). Unconscious perception: A model-based approach to method and evidence. Perception \& Psychophysics, 66, 846-867.

Snow, J. C., \& MatTingley, J. B. (2006). Goal-driven selective attention in patients with right hemisphere lesions: How intact is the ipsilesional field? Brain, 129, 168-181. doi:10.1093/brain/awh690

Vorberg, D., Mattler, U., Heinecke, A., Schmidt, T., \& SchwarzвАCH, J. (2003). Different time courses for visual perception and action priming. Proceedings of the National Academy of Sciences, 100, 6275-6280. doi:10.1073/pnas.0931489100

\section{NOTES}

1. Some of the data reported in this article were presented at the 41 st Annual Meeting of the Psychonomic Society in New Orleans (Ro \& Singhal, 2000).

2. Although it is impossible to determine whether our neutral stimulus was truly neutral (see Jonides \& Mack, 1984), we composed a neutral stimulus that was the sum of the CRT phosphor values of the two target stimuli. Given our previous work demonstrating that unconscious priming occurs at wavelength-dependent levels of processing (Breitmeyer, Ro, \& Singhal, 2004), this composition of the neutral stimulus seemed reasonable for determining whether unconscious priming is facilitatory or inhibitory in nature.

3. Only participants with at least 64 total trials in the congruent, aware condition were included in this analysis.

4. It could also be argued that on the "unaware" trials, participants may have been aware of the disk at the time of the color response to the annulus, but that this awareness may have faded from memory by the time a response was made regarding the presence or absence of the disk. Although we cannot completely rule out this possibility, we find it unlikely for two reasons. First, the participants were well informed that the task was to first make a speeded report on the color of the annulus, then to simply report whether a disk was perceived on that given trial. Thus, any information regarding the presence of the disk was to be explicitly held in memory until probed for that response less than $1 \mathrm{sec}$ later. This makes forgetting unlikely. Second, if participants were in fact aware of the disk on the "unaware" trials, their performance should have been similar to that on the aware trials in this experiment, which clearly was not the case.

5. This change in analysis procedures was necessary because the equipment used to collect the data for this experiment only provided the mean RTs with these cutoffs. The rationale for using an upper RT limit of $900 \mathrm{msec}$ was based on prior pilot studies showing that individual observers seldom, if ever, generated mean RTs longer than $500 \mathrm{msec}$. With standard deviations averaging around $30 \mathrm{msec}$, an individual RT of $900 \mathrm{msec}$ would lie in excess of $10 S D$ s from a typical mean RT. We also performed a reanalysis of the data from Experiment 1 using cutoffs similar to those used in this experiment, and found effects similar or identical to the standard deviation outlier removal procedure.

6. The one exception to this is Experiment 4 of Klotz and Neumann (1999), in which awareness was assessed on a trial-by-trial basis, as in our Experiment 1; but their results were not binned and analyzed according to the awareness responses.

7. The degree of interference between the "aware" and "unaware" conditions is not directly comparable in some of these experiments. In addition to differing physical conditions that can produce awareness of an event, such as the SOA between the disk and the annulus, there are also numerous cognitive factors that can lead to an "aware" percept of an otherwise near-threshold stimulus. Thus, all of our discussions regarding the degree of interference between aware and unaware distractors in this article reflect a shorthand way of describing differing general cognitive states that may contribute to the magnitude of response channel activation.

(Manuscript received July 10, 2008; revision accepted for publication July 31, 2008.) 